Mon. Not. R. Astron. Soc. 000, 1 4(2017) Printed 15 October $2018 \quad$ (MN LTEX style file v2.2)

\title{
Comparison of Gaia and asteroseismic distances
}

\author{
M. Yıldız ${ }^{1 \star}$, Z. Çelik Orhan ${ }^{1}$, S. Örtel ${ }^{1}$ and M. Roth ${ }^{2}$ \\ ${ }^{1}$ Department of Astronomy and Space Sciences, Science Faculty, Ege University, 35100, Bornova, İzmir, Turkey \\ ${ }^{2}$ Kiepenheuer-Institut für Sonnenphysik, Schöneckstr. 6, D-79104 Freiburg, Germany
}

Accepted 2016 May 15. Received 2016 April 11; in original form 2016 April 11

\begin{abstract}
Asteroseismology provides fundamental properties (mass, radius and effective temperature) of solar-like oscillating stars using so-called scaling relations. These properties allow the computation of the asteroseismic distance of stars. We compare the asteroseismic distances with the recently released Gaia distances for 74 stars studied in Yıldiz et al. There is a very good agreement between these two distances; for 64 of these stars, the difference is less than 10 per cent. However, a systematic difference is seen if we use the effective temperature obtained by spectroscopic methods; the Gaia distances are about 5 per cent greater than the asteroseismic distances.
\end{abstract}

Key words: stars: distance - stars: evolution - stars: fundamental parameters - stars: interiors - stars: late-type - stars: oscillations.

\section{INTRODUCTION}

The distance of stars is one of the major astrophysical parameters, whose precise measurement will shed significant light on stellar evolutionary theory, and the chemical enrichment of the Milky Way and planetary systems. The Hipparcos mission (Perryman et al. 1997; van Leeuwen 2007) allowed a detailed investigation of stars nearer than $100 \mathrm{pc}$ to us. We expect to be able to increase this distance at least 10-fold as a result of the recent Gaia mission (Gaia Collaboration et al. 2016b), with much smaller uncertainty. In this study, the Gaia distances ( $d_{\text {GAIA }}$ ) are compared with the so-called asteroseismic distances $\left(d_{\text {sis }}\right)$ computed from oscillation frequencies of the solar-like oscillating stars studied in Yildiz et al. (2016).

Highly precise parallaxes $(\pi)$ of over one billion stars will be obtained by the ESA Gaia mission (Gaia Collaboration et al. 2016b). The duration of the mission will be at least $5 \mathrm{yr}$, and the most accurate data on position, proper motion and parallax of stars brighter than 20.7 mag will be released in 2022. The mission, launched in 2013, released data (DR1) on measurements taken within the first 14 months of observations for more than two million stars (Gaia Collaboration et al. 2016a) which are saved in the Tycho-2 catalogue (Høg et al. 2000). The astrometric data is available for 74 of 89 target stars in Y1ldiz et al. (2016).

Asteroseismology is currently experiencing a golden age with the space-based missions Kepler (Borucki et al. 2010) and CoRoT (Baglin et al. 2006 ). TESS (to be launched in 2018, Sullivan et al. 2015) and PLATO (to be launched in 2025; Catala et al. 2011) will also further the scientific outcomes in this field. Asteroseismology

\footnotetext{
* E-mail: mutlu.yildiz@ege.edu.tr
}

is leading to great advances in the precision of the internal structure model of the solar-like oscillating stars in particular (Chaplin $\&$ Miglio 2013). The mass $(M)$ and radius $(R)$ of these stars can be found from scaling relations that relate $M$ and $R$ to the mean of so-called large separation between oscillation frequencies $(\langle\Delta \nu\rangle)$, frequency of maximum amplitude $\left(\nu_{\max }\right)$ and effective temperature ( $\left.T_{\text {eff }}\right)$. The compressibility (the first adiabatic exponent, $\Gamma_{1 \mathrm{~s}}$ ) at the surface of these stars had until recently been taken as constant in the derivation of these relations (Kjeldsen \& Bedding 1995). However, Yildiz et al. (2016) have shown that this is not the case. After obtaining new scaling relations based on modifications to conventional ones, they achieved results in very good agreement with non-asteroseismic predictions, for example, for Procyon A (Aufdenberg, Ludwing \& Kervella 2015; Bond et al. 2015).

The radius is required to compute the luminosity of a star. The modified scaling relation for stellar radius in solar units $\left(R_{\text {sca }} / \mathrm{R}_{\odot}\right)$ is given by Yildız et al. (2016) as

$$
\frac{R_{\text {sca }}}{\mathrm{R}_{\odot}}=\frac{\left(\nu_{\max } / \nu_{\max \odot}\right)}{(\langle\Delta \nu\rangle /\langle\Delta \nu \odot\rangle)^{2}}\left(\frac{T_{\text {eff }}}{T_{\text {eff }} \odot} \frac{\Gamma_{1 \mathrm{~s}} \odot}{\Gamma_{1 \mathrm{~s}}}\right)^{1 / 2} \frac{f_{\Delta \nu}^{2}}{f_{\nu}},
$$

where $f_{\nu}$ is the ratio of $\nu_{\max }$ to acoustic cut-off frequency and $f_{\Delta \nu}$ is defined as the ratio of $\langle\Delta \nu\rangle /\left\langle\Delta \nu_{\odot}\right\rangle$ to square root of mean density in solar units $\left(\sqrt{\rho / \rho_{\odot}}\right)$. In conventional scaling relations, $f_{\Delta \nu}$ and $f_{\nu}$ are equal to 1 . These are clearly functions of $\Gamma_{1 \mathrm{~s}}$ (see equations 7 and 11, in Y1ldiz et al. 2016).

This Letter is organized as follows: Section 2 presents the method for the computation of asteroseismic distance. Section 3 is devoted to the results and their comparison. Finally, in Section 4, conclusions are drawn. 


\section{ASTEROSEISMIC AND Gaia DISTANCES}

In order to determine the distance of a star from distance modulus, its luminosity must be found. Then, its $T_{\text {eff }}$ and $R$ are required. $T_{\text {eff }}$ can be determined using spectroscopic and photometric methods, in addition to entirely new asteroseismic methods developed by Y1ldiz et al. $(2014,2016)$ for the solar-like oscillating stars. However, the most precise prediction of asteroseismology is for radius; $R$ is given in terms of $\nu_{\max },\langle\Delta \nu\rangle$ and $T_{\text {eff }}$. The radii of the target stars are computed from equation (1). For $\Gamma_{1 \mathrm{~s}}$, we use the expression given by Yildiz et al. (2016) in terms of $T_{\text {eff }}$ :

$$
\frac{1}{\Gamma_{1 \mathrm{~s}}}=1.6\left(\frac{T_{\mathrm{eff}}}{T_{\mathrm{eff} \odot}}-0.96\right)^{2}+0.607 .
$$

From the luminosity $(L)$ of a star, we evaluate its bolometric magnitude. Bolometric corrections for the target stars are computed from Lejeune, Cuisinier \& Buser's (1998) tables using observed $[\mathrm{Fe} / \mathrm{H}]$ and $T_{\text {eff }}$ from spectroscopy and asteroseismology. Following this, we obtain the absolute magnitude from the bolometric magnitude and correction, and the distance modulus from the observed visual magnitude $(V)$ and absolute magnitude. In our computations, we take the spectroscopic effective temperature $\left(T_{\mathrm{eS}}\right)$ as $T_{\text {eff }}$ of the target stars. For testing the effect of $T_{\text {eff }}$ on $d_{\text {sis }}$, we also compute $d_{\text {sis } 0}$ from the asteroseismic effective temperature, which is computed from the oscillation frequency of min0 (equation 16 in Y1ldiz et al. 2016).

We directly compute distances from parallaxes given by Gaia $\left(\pi_{\text {GAIA }}\right)$ and Hipparcos $\left(\pi_{\text {HIP }}\right)$. The Gaia parallaxes are available for 74 of the stars studied in Y1ldiz et al. (2016). With the exception of one red giant (HD 181907/HIP 95133), all are main-sequence and sub-giant stars.

The uncertainty of $d_{\text {sis }}$ is computed from the uncertainties in $T_{\text {eff }}, R_{\text {sca }}$ and $V$. Luminosity is perhaps the most uncertain parameter among the fundamental stellar parameters. Its uncertainty can be computed from uncertainties of radius and $T_{\text {eff }}$ in quadrature:

$$
\frac{\Delta L}{L}=\sqrt{\left(2 \frac{\Delta R}{R}\right)^{2}+\left(4 \frac{\Delta T_{\text {eff }}}{T_{\text {eff }}}\right)^{2}} .
$$

The typical uncertainty in asteroseismic distance is computed by taking the luminosity as $L^{\prime}=L+\Delta L$. Using $L^{\prime}$ in place of $L$, we obtain the most uncertain distance $\left(d_{\mathrm{sis}}^{\prime}\right)$. The difference between $d_{\text {sis }}^{\prime}$ and $d_{\text {sis }}\left(\Delta d_{\text {sis }}^{\prime}\right)$ is the typical uncertainty in asteroseismic distance. We also take into account the uncertainty of $V$ in the computation of $d_{\text {sis }}^{\prime}$. Uncertainty of $\pi$ is computed from $\Delta d_{\text {sis }}^{\prime}$ :

$$
\frac{\Delta \pi_{\mathrm{sis}}}{\pi_{\mathrm{sis}}} \approx \frac{\Delta d_{\mathrm{sis}}}{d_{\mathrm{sis}}},
$$

where the second and higher terms are neglected. However, these terms may play a dominating role when the relative errors are larger than about 10 per cent.

\section{RESULTS AND DISCUSSIONS}

In Fig. 1 , the asteroseismic parallax $\left(\pi_{\text {sis }}\right)$ computed from distance modulus is plotted against to $\pi_{\mathrm{GAIA}} . d_{\mathrm{GAIA}}$ of the targets range from 21 pc (16 Cyg A/HIP 96895 and 16 Cyg B/HIP 96901) to 433 pc (KIC 10920273/TYC 3547-1968-1). For comparison, $\pi_{\text {HIP }}$ is also plotted. The greatest difference between $\pi_{\text {sis }}$ and $\pi_{\text {GAIA }}$ occurs for KIC $8379927 / \mathrm{HIP} 97321\left(\pi_{\mathrm{GAIA}}=18.76\right.$ mas $)$. Its $\pi_{\mathrm{HIP}}$ ( 24.86 mas) is closer to $\pi_{\text {sis }}\left(29.18\right.$ mas) than its $\pi_{\text {GAIA }}$. There is in general a very good agreement between $\pi_{\text {sis }}$ and $\pi_{\text {GAIA }}$ for the

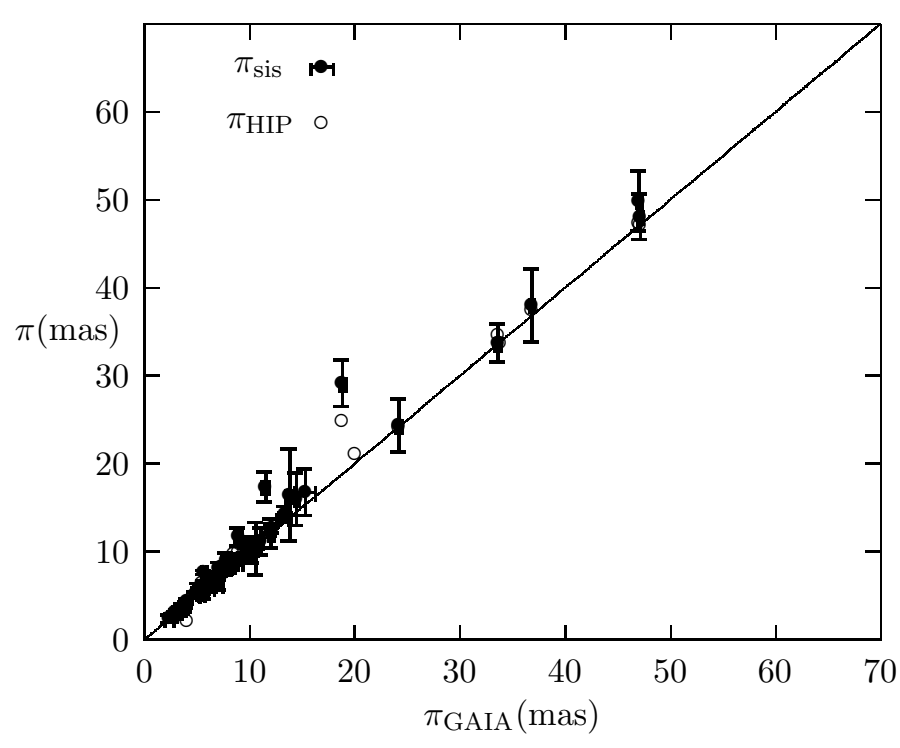

Figure 1. Asteroseismic parallax (filled circle) is plotted with respect to the Gaia parallax in units of mas. For comparison, the Hipparcos parallax (circle) is also plotted if available. For one of the stars, namely KIC $7341231 / \mathrm{HIP} 92775, \pi_{\mathrm{HIP}}\left(d_{\mathrm{HIP}}=490 \mathrm{pc}\right)$ is extremely uncertain but its $\pi_{\mathrm{GAIA}}$ and $\pi_{\mathrm{sis}}$ are in good agreement: $d_{\mathrm{GAIA}}=249 \mathrm{pc}, d_{\mathrm{sis}}=230 \mathrm{pc}$.

majority of the targets. However, there is a systematic difference between $d_{\text {sis }}$ and $d_{\text {GAIA }}$. The latter is about 5 per cent greater than the former. The scatter of the measurement difference is calculated by determining the unit-weight standard deviations, i.e. incorporating per measurement the various error contributions. The variance is $2.01 \mathrm{mas}^{2}$ for $N=74$. Excluding the largest two values the variance does not change significantly and is $1.93 \mathrm{mas}^{2}$.

The same difference is also seen between $d_{\text {sis } 0}$ computed

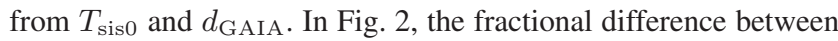
$d_{\text {sis }}$ and $d_{\text {GAIA }}$ is plotted with respect to the $V$ magnitude of the target stars. For most of the targets, the difference between these two distances is less than 5 per cent; $\delta d / d_{\text {GAIA }}=\mid d_{\text {sis }}-$ $d_{\text {GAIA }} \mid / d_{\text {GAIA }}<0.05$; however, for five stars, KIC $1435467 /$ TYC 2666-333-1, KIC 6933899/TYC 3128-1911-1, KIC 8379927/HIP 97321, KIC 9025370/HIP 97321 and KIC 10454113/HIP 97321, the difference is relatively large. With the exclusion of these untypical stars, the difference slightly increases with respect to $V$ when $V>8.3 \mathrm{mag}$, as shown by the solid lines in Fig. 2 .

For 64 of 89 stars, the difference between $d_{\text {sis }}$ and $d_{\text {GAIA }}$ is less than 10 per cent. Silva-Aguirre et al (2012) found the difference between $d_{\text {sis }}$ and $d_{\text {HIP }}$ for 22 stars less than 20 per cent.

If we assume that a small discrepancy is due to an uncertainty in $d_{\text {sis }}$, there are two possible reasons: uncertainties in $R$ and $T_{\text {eff }}$. If due to radius, equation (1) underestimates the radius of stars by about 5 per cent, and if due to $T_{\text {eff }}, T_{\mathrm{eS}}$ is found to be 2 per cent less than the $T_{\text {eff }}$ of the targets, or equivalently $120 \mathrm{~K}$ less.

If we compare $d_{\mathrm{sis} 0}$ with $d_{\mathrm{GAIA}}$, we find the same mean fractional difference, about 0.05 . However, the difference between $d_{\text {sis } 0}$ and $d_{\mathrm{GAIA}}$ is less than 10 per cent for 46 of the targets.

In order to better compare the agreement of the two measurements, Fig. 3 displays the difference $\delta \pi=\pi_{\text {GAIA }}-\pi_{\text {sis }}$ versus the mean out of both measurements $\bar{\pi}=\left(\pi_{\text {sis }}+\pi_{\text {GAIA }}\right) / 2$ in the form of a Bland-Altman diagram (Bland \& Altman 1999). Based on this scatter plot, we find a positive bias, i.e. the Gaia measurements give, on average, smaller parallaxes than the seis- 
Comparison of Gaia and asteroseismic distances

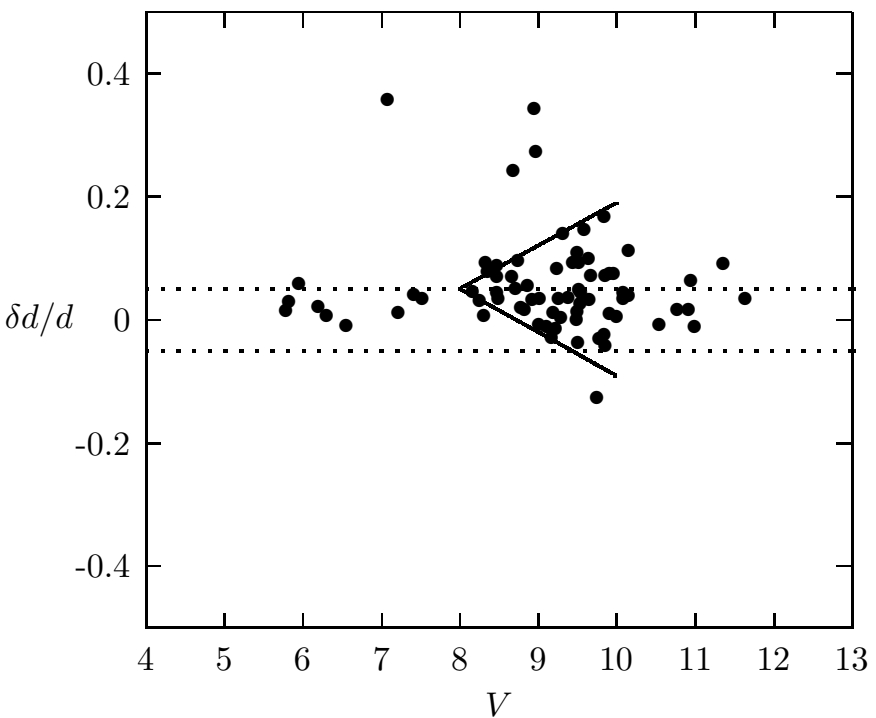

Figure 2. Fractional difference between the asteroseismic distance and the Gaia distances of the targets is plotted with respect to $V$. The dotted lines show a 5 per cent uncertainty level. For five stars, the difference is very great. The solid lines represent an increase in uncertainty for the stars with $V>8.3 \mathrm{mag}$

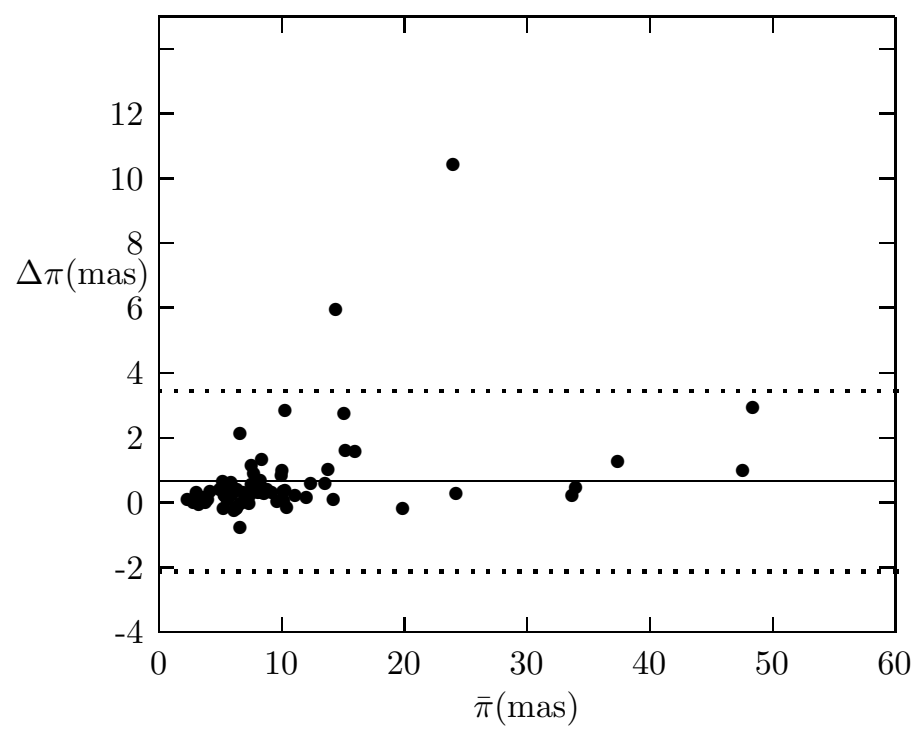

Figure 3. Bland-Altman plot of the difference $\delta \pi=\pi_{\text {sis }}-\pi_{\text {GAIA }}$ between the asteroseismic and the Gaia parallaxes of the Kepler targets versus the mean parallax $\left(\bar{\pi}=\left(\pi_{\text {sis }}+\pi_{\mathrm{GAIA}}\right) / 2\right)$ of these two measurements. The solid horizontal line shows $\overline{\delta \pi}=0.67$ mas, and the dotted horizontal lines represent $\overline{\delta \pi} \pm 1.96 \operatorname{std}(\delta \pi)$ with $\operatorname{std}(\delta \pi)=1.82$ mas for the Hipparcos parallaxes.

mic measurements. The mean of the difference is $\delta \pi=0.67$ mas $(\delta d=6.9 \mathrm{pc})$. The scatter of the measurement difference $\operatorname{var} \delta \pi=$ $\sum(\delta \pi-\overline{\delta \pi})^{2} /(\mathrm{N}-1)$ is $2.01 \mathrm{mas}^{2}$ for $N=74$. Excluding the largest two values, the variance is reduced to $1.93 \mathrm{mas}^{2}$. Based on the assumption that the distribution is Gaussian, $95 \%$ of the values lie between the values $\bar{\pi} \pm 1.96 \operatorname{std}(\delta \pi)=[-2.11,3.45]$ mas for $N=74$ or $[-2.27,3.18]$ mas for $N=72$, respectively. Given the

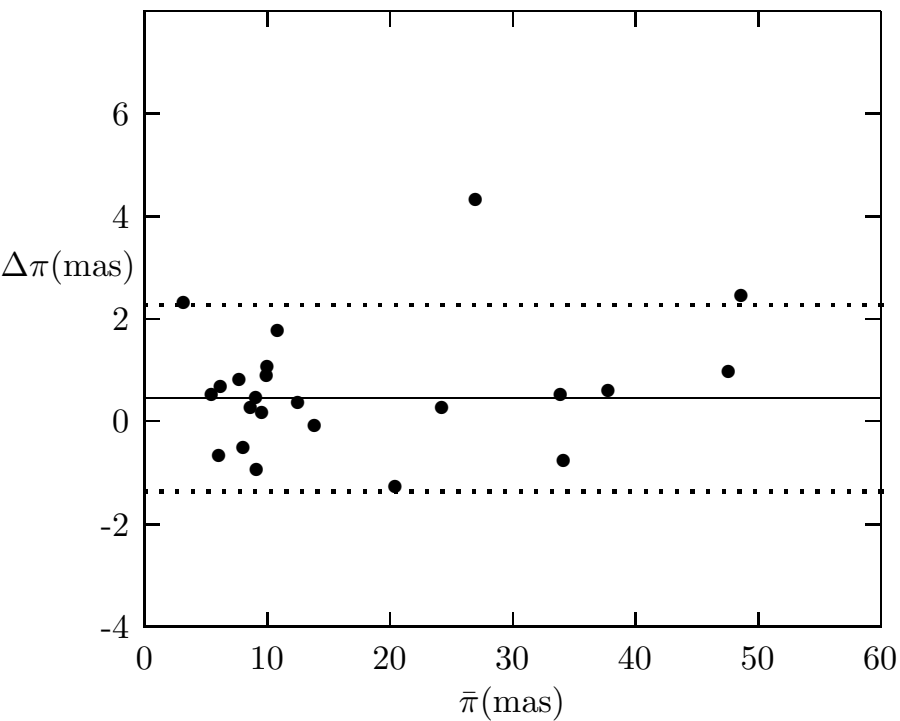

Figure 4. Same as Fig. 3 but the Hipparcos parallaxes are used in place of the Gaia parallaxes. $\overline{\delta \pi}=0.46$ mas and $\operatorname{std}(\delta \pi)=3.31$ mas for the Hipparcos parallaxes.

data the difference and the scatter do not depend on the magnitude of the measurements itself.

Fig. 4 displays a Bland-Altman diagram for comparing $\pi_{\text {sis }}$ with the Hipparcos $\pi_{\text {HIP }}$ parallax measurements. Here a bias cannot be detected on the basis of the data set, but there are indications that the scatter, i.e. the variance of the difference, increases with larger distances. However, this finding might still be a result that is due to the small size of the sample.

The mean difference between $\pi_{\mathrm{HIP}}$ and $\pi_{\mathrm{GAIA}}$ is 0.47 mas. This shows that there are very similar differences between three different parallaxes. Therefore, it is very difficult to find the sources of uncertainties. However, uncertainties in $T_{\text {eff }}$ and $\nu_{\max }$ might be the main source for the uncertainty of $\pi_{\text {sis }}$.

\section{CONCLUSIONS}

For solar-like oscillating stars, we can determine $M, R$ and even $T_{\text {eff }}$ using the new scaling relations given by Y1ldiz et al. (2016). This allows the computation of asteroseismic distances by using bolometric correction and observed $V . d_{\text {sis }}$ is computed for the stars studied in Y1ld1z et al. (2016). For 74 of these stars, the Gaia distance is also available. There is a very good agreement between $d_{\text {sis }}$ and $d_{\text {GAIA }}$, the distances that vary by less than 10 per cent for 64 stars. However, there is a systematic difference between these two distances: $d_{\text {GAIA }}$ is 5 per cent greater than $d_{\text {sis }}$. This systematic difference does not depend on the magnitude of the distance. Possible sources of uncertainty in $\pi_{\text {sis }}$ are due to uncertainties in $T_{\text {eff }}$ and $\nu_{\max }$. We hope that more precise Gaia parallaxes to be released in the near future will enlighten the situation.

\section{ACKNOWLEDGEMENTS}

This work is supported by the Scientific and Technological Research Council of Turkey (TÜBİTAK: 112T989). MR acknowledges support from the European Research Council under the Eu- 
ropean Union's Seventh Framework Program (FP/2007-2013)/ERC Grant Agreement no. 307117.

\section{REFERENCES}

Aufdenberg J. P., Ludwing H. G., Kervella P., 2015, ApJ, 633, 424 Baglin A., Michel E., Auvergne M., COROT Team, 2006, in Fridlund M., Baglin A., Lochard J., Conroy L., eds, ESA Special Publication Vol. 1306, ESA Special Publication. p. 33

Bland J. M., Altman D. G., 1999, Stat. Methods. Med. Res., 8, 135

Bond H. et al, 2015, ApJ, 813, 2

Borucki W. J., Koch D., Basri G., et al. 2010, Science, 327, 977

Catala C., Appourchaux T., Plato Mission Consortium, 2011, J. Phys. Conf. Ser., 271, 012084

Chaplin W. J., Miglio A., 2013, ARA\&A, 51, 353

Gaia Collaboration, Brown A. G. A., Vallenari A., et al. 2016a, A\&A, doi:10.1051/0004-6361/201629512

Gaia Collaboration, Prusti T., de Bruijne J. H. J., et al. 2016b, A\&A, doi:10.1051/0004-6361/201629272

Høg E. et al., 2000, A\&A, 357, 367

Kjeldsen H., Bedding T. R., 1995, A\&A, 293, 87

Lejeune T., Cuisinier F., Buser, R., 1998, A\&AS, 130, 65

Perryman M. A. C. Lindegren L., Kovalevsky J., et al. 1997, A\&A, 323, 49

Silva Aguirre V. et al., 2012, ApJ, 757, 99

Sullivan P. W. et al., 2015, ApJ, 809, 77

van Leeuwen F., 2007, A\&A, 474, 653

Yıldız M., Çelik Orhan Z., Aksoy Ç., Ok S., 2014, MNRAS, 441, 2148

Yıldız M., Çelik Orhan Z., Kayhan C., 2016, MNRAS, 462, 1577 